\title{
Lapsus de Laïus
}

Entre régicide et parricide, l'introuvable meurtre du père

Lapsus of Laïus. Either Regicide or Parricide, the Indefinable "Murder of the Father"

斌父一一匿跡于斌君與斌親之間

\section{Jérôme Bourgon}

\section{(2) OpenEdition}

\section{Journals}

Édition électronique

URL : http://journals.openedition.org/extremeorient/229

DOI : 10.4000/extremeorient.229

ISSN : 2108-7105

Éditeur

Presses universitaires de Vincennes

\section{Édition imprimée}

Date de publication : 1 janvier 2012

Pagination : 313-339

ISSN : 0754-5010

\section{Référence électronique}

Jérôme Bourgon, «Lapsus de Laïus », Extrême-Orient Extrême-Occident [En ligne], Hors-série | 2012, mis en ligne le 01 janvier 2015, consulté le 19 avril 2019. URL : http://journals.openedition.org/ extremeorient/229; DOI : 10.4000/extremeorient.229 


\title{
Lapsus de Laïus. Entre régicide et parricide, l'introuvable meurtre du père
}

\author{
Jérôme Bourgon
}

Peut-on siniser Edipe? Les psychanalystes qui prospèrent à présent en Chine l'ont fait à leur manière, en traduisant son supposé complexe par une expression aussi charmante qu'approximative: le complexe de «désirer» ou «aimer sa mère» (lianmu qingjie ou aimu qingjie), tandis que d'autres préfèrent employer la transcription phonétique de Edipus : «le complexe de Yi-tipou-sse» (Yidipusi). C'est un dilemme inhérent à la vaste entreprise de traduction des termes occidentaux qui a accompagné la modernisation chinoise : traduire le sens, en acceptant de le trahir quelque peu, ou ne conserver que la prononciation, en courant le risque que la signification du terme ne soit accessible qu'aux seuls initiés. Va pour le «complexe de désirer sa mère» ou «de Yi-di-pu-si», mais n'aurait-on oublié personne? Le complexe ne consisterait-il qu'à trop aimer sa mère ? N'y aurait-il ici pas quelque dégât collatéral à déplorer? Quid de Laïos, alias Laïus, roi de Thèbes, père et victime d'Edipe ${ }^{1}$ ? Tant qu'à traduire le sens, ne pouvait-on créer le «complexe-d'aimer-sa-mère-et-tuer-son-père », ce qui en chinois n'aurait nécessité que deux caractère supplémentaires (lianmu shafu qingjie)?

Lapsus forcément révélateur que cette disparition du roi-père, qui laisse ouvert - ou boiteux, comme l'étaient CEdipe et Laïus - le fameux « triangle œdipien ». Mais révélateur de quoi au juste ? Plutôt qu'une censure dictée par l'inconscient des traducteurs, ou qu'un impensé lové dans les replis du langage, nous $\mathrm{y}$ verrons un indice que le meurtre du père n'a pas dans la tradition chinoise la signification qu'il a dans la tradition occidentale. Ce qui suggère bien sûr des

1. On adopte à présent la transcription Laïos, de préférence à Laïus qui était usité au XIX ${ }^{e}$ siècle. Laïus est devenu le nom commun d'un discours interminable après qu'au concours d'entrée à Polytechnique de 1804 eut figuré le libellé du sujet suivant: «Imaginez la réponse de Laïus à CEdipe», ce qui suscita chez les candidats des flots d'éloquence intarissables! 
différences dans le statut et la nature de l'autorité paternelle. Voilà d'ailleurs le véritable objet de notre investigation : la figure, le statut et l'autorité du père en Chine et en Occident, le meurtre ayant pour seul intérêt de tirer les choses au clair, s'il est permis de s'exprimer ainsi.

Il ne s'agit donc pas de recenser des exemples de fils tuant leur père, ni de spéculer sur un acte qu'il n'y a pas lieu de supposer plus fréquent ici qu'ailleurs ; encore moins nous interrogerons-nous sur les désirs conscients ou inconscients des acteurs. Notre propos est ouvertement «dogmatique» puisqu'il consiste à interroger la fonction dogmatique qui vertèbre le système de valeurs de la civilisation chinoise: par «dogmatique», il faut entendre le discours qui ordonne le donné biologique, et son fondement, la reproduction sexuée ainsi que les relations qui en découlent, en institutions durables, orientées vers un centre, idéalement l'État ${ }^{2}$. L'activité dogmatique consiste principalement à créer des généalogies, qui transforment la génération biologique en institutions légitimes. En reliant à un ancêtre fondateur un clan, une dynastie, ou l'empire lui-même, la dogmatique crée des fictions de légitimité. La Chine est à cet égard une création dogmatique remarquable par sa longévité et son ampleur ${ }^{3}$.

Les sociétés ont engendré bien des ordres dogmatiques différents, dont la typologie reste d'ailleurs à faire. Deux suffiront à notre propos. Le premier est le droit gréco-romain perpétué jusqu'à nous par l'Église catholique au travers du droit canon, qui fournit le cadre dans lequel nous concevons spontanément le parricide et le meurtre du père. Le second est ce qu'il est convenu d'appeler, faute de mieux, «le confucianisme», mais nous verrons qu'il s'agit en fait du courant d'interprétation des Classiques en «caractères modernes » (jinwen), qui est précisément le plus dogmatique. Notre société est aussi éloignée qu'il est possible de celle des jurisconsultes romains ou des Pères de l'Église, de même que la Chine actuelle s'est éloignée de celle de Confucius, mais paternité et filiation restent toutefois définies dans des termes remarquablement stables. Les révolutions modernes, de la protestante à la bolchévique, ont ébranlé sans le supprimer l'échafaudage généalogique, la mesure de proximité ou d'éloignement et, par voie de conséquence, le cercle où s'exerce l'interdit de l'inceste, les règles de transmission du nom et de succession au patrimoine - car Calvin, Robespierre ou Mao restent fils de leur père comme leur nom l'indique, et la seule ruse admise, celle du pseudonyme à la

2. Telle que définie par Pierre Legendre, «[1]a fonction dogmatique consiste, dans une société, à fonder et mettre en scène la fonction biologique de la reproduction. Il s'agit, en signifiant des raisons de vivre et de mourir, de soutenir la cause humaine au moyen d'institutions » (Leçons II : 51).

3. Très éclairant de ce point de vue est le lien organique des clans cantonais avec la dynastie régnante, mis en lumière par Faure (2007): 2 et $125 s q$. 
Lénine-Oulianov, ne trompe personne ${ }^{4}$. Voilà le cadre au sein duquel le parricide prend son sens de crime «contre nature», avant d'apparaître au dénouement des tragédies ou à la rubrique des faits divers. C'est de ce père-là, créature fictive du discours dogmatique, qu'il est question dans le parricide.

Le droit est l'activité pratique et savante qui prend en charge la fonction dogmatique. Sans en être l'expression unique, il en est la plus constante et la plus pragmatique. Car enfin, c'est une chose de définir l'inceste ou le parricide à partir des règles rituelles, et c'en est une autre que de condamner, punir et exécuter l'individu incestueux ou parricide. Le juge y risque son confort intellectuel, voire son Salut; les rites et les croyances y sont soumis à l'épreuve du sang, les attendus du jugement doivent convaincre les foules que le supplice qui se déroule sous leurs yeux est légitime. C'est pourquoi la peine du parricide nous dit tant sur la nature du crime, et les valeurs qui la fondent. Il faudra donc comparer les peines pratiquées en Chine et en Occident, et déchiffrer le message dogmatique qu'elles recèlent. Tout autant, il faudra s'intéresser à la position du crime dans la nomenclature des crimes connexes: dans les deux civilisations de référence, le meurtre du père voisine avec le régicide, d'une part, et d'autre part avec le parricide, entendu dans une acception extrêmement étendue, englobant les parents les plus éloignés, voire l'ensemble du genre humain.

Là gît pourtant la différence que nous chercherons à mettre en lumière : alors qu'en Occident, le meurtre du père constitue l'essence du parricide, le modèle du crime contre nature, sur lequel se déclinent par extension ou métaphore les autres crimes (régicide, meurtre d'autres parents), en Chine, le meurtre du père n'est rien en lui-même: soit c'est un régicide, l'assassinat d'un souverain qui se trouve être aussi un père; soit le père se trouve confondu avec bien d'autres parents dans la définition du crime de parricide, de sorte que son assassinat n'est pas plus grave que celui d'un aïeul de la lignée maternelle. Entre la fonction politique - le souverain - et la chaîne de reproduction de la parenté, il semble qu'il n'y ait pas de place distincte pour l'autorité paternelle, au sens romain et, par dérivation, occidental du terme. Plus déroutant encore, l'autorité qui nous semble unilatéralement paternelle et, partant, masculine, semble en Chine combiner le paternel et le maternel, le masculin et le féminin. Voyons cela de plus près.

\section{$S h i$ 斌: le régicide-parricide, attentat contre l'autorité publique}

Le terme de parricide n'a pas d'équivalent exact dans la tradition chinoise; plusieurs termes lui correspondent plus ou moins, dont nous examinerons ceux

4. Ceci pour ne rien dire de la révolution libérale en cours, qui s'attaque bien plus que ses devancières aux fondations de l'ordre généalogique. 
qui nous semblent les plus pertinents. Le terme shi est celui qui présente les plus grandes affinités avec le «crime public » de parricide tel que le concevait la tradition romaine. On en trouve une définition très suggestive dans le Bohutong, une compilation de notes prises au cours de discussions sur les Classiques qui auraient eu lieu en 79 ap. J.-C. ${ }^{5}$. Le Classique le plus souvent cité est le Chunqiu, ces Annales ou Chronique des Printemps et Automnes de l'État de Lu, patrie de Confucius, qui s'achèvent par l'annonce de sa venue. Plus précisément, le texte assez obscur des Annales y est décrypté à la manière du commentaire de Gongyang Gao. Nous avons là affaire à une sorte de vulgate de la tradition des textes dits «en caractères modernes », le courant dominant lors de la formation de l'orthodoxie confucéenne sous la dynastie des Han. Et c'est fort bien, car il s'agit là d'un confucianisme parfois qualifié de «légiste», tant la fonction dogmatique de l'orthodoxie confucéenne laisse encore apparaître dans toute leur crudité ses éléments premiers : généalogie, filiation, parricide, usurpation et vengeance.

Voici comment le Bohutong définit le terme shi:

Qu'appelle-t-on shi1? shi(1), c'est shi(2): «essayer, tâter le terrain». Cela veut dire que quand un sujet ou un fils [décide de] tuer son souverain ou son père, il n'ose achever [son projet d'un coup], il attend l'occasion de passer à l'acte, en préparant pas à pas son régicide ou son parricide. Le Livre des Mutations dit: quand un sujet tue son souverain, quand un fils tue son père, c'est pour une cause qui n'est pas née le jour même [mais qui remonte à loin] ${ }^{6}$.

Voici donc deux couples opposés et complémentaires: le premier est composé de jun et chen, le souverain et son sujet. En réalité, ce «sujet» est un ministre, un féal, un subordonné direct, qui entretient une relation personnelle avec son supérieur. Délégué du pouvoir du jun, le chen est en position de l'usurper et de se débarrasser de son titulaire. Le second couple, composé de fu et $z i$, le père et le fils, n'existe en fait que comme l'ombre portée du précédent, placés qu'ils sont dans la même

5. Ban Gu, l'auteur de l'histoire officielle des Han ou Hanshu, est couramment donné comme son auteur, le Hanshu citant d'ailleurs un décret impérial ordonnant à Ban $\mathrm{Gu}$ d'effectuer une compilation des débats tenus dans le Hall du Tigre Blanc (Bohu guan). Le lien avec l'ouvrage existant n'en est pas évident pour autant, car les noms des orateurs cités ne sont pas les mêmes. Qui plus est, l'ouvrage se voit mentionné sous plusieurs titres, qui varient d'un auteur et d'une période à l'autre, sans que l'on sache bien si ces mentions diverses se réfèrent au même texte. Ce dernier nous est parvenu dans une édition de 1305, révisée par Lu Wenchao au XVIII ${ }^{e}$ siècle. Il comporte de multiples interpolations, qui suscitent des commentaires excédés de certains spécialistes, selon lesquels «rien n'est jamais clair dans le Bohutong », lequel «mélange tout», mais l'on sait aussi que les érudits chinois sont rarement satisfaits de leurs sources!

6. Bohutong, 99, «Zhufa 誅伐». 
situation d'usurpation potentielle. Les deux usurpations portent le même nom de $s h i$, qui est explicité par le biais d'une étymologie douteuse mais évocatrice: la composante phonétique shi 式 commune aux deux caractères autorise un rapprochement entre le régicide parricide et un autre terme, shi (2) 試, qui signifie «examiner, tester, essayer». Le crime est ainsi envisagé comme «tentative»-nous y reviendrons un peu plus loin. Voilà ce que l'on peut dire à partir d'une traduction qui s'efforce de répondre aux règles de la grammaire et aux exigences de la logique. La syntaxe chinoise autorise toutefois des effets de parallélisme que la version suivante tente de restituer:

Quand le sujet-fils tue son souverain-père, il n'ose achever son projet d'un coup, mais attend l'occasion de passer à l'acte et prépare pas à pas son régicide-parricide.

Le binôme sujet-fils (chen zi) doit se comprendre comme formant un terme unique, et il en va de même du binôme souverain-père ( $j u n f u$ ). Chacun de ces binômes forme le pôle d'une relation de pouvoir - pouvoir pur, relation abstraite du lien particulier qui l'a causée. Le procédé rappelle ces sentences parallèles que les Chinois accrochent de part et d'autre des autels et autres lieux sacrés. Il s'agit là de deux phrases construites sur le même modèle, et dont chaque mot - ou chaque caractère - trouve son correspondant dans la phrase en vis-à-vis, avec la même fonction grammaticale. Les phrases se correspondent ainsi terme à terme sur le plan sémantique et syntaxique. En vérité, le sens ultime de ces formules ne réside dans aucune des deux propositions, mais dans le rapport qu'elles entretiennent, de même que la vision binoculaire restitue la réalité dans ses trois dimensions. Le parallélisme fils-sujet/père-souverain, et sa mise en péril par le même crime de $s h i$, fait ressortir la figure sacrée, la place abstraite de l'autorité comme telle. Idée banale, mais technique superbe! Tout l'art dogmatique est dans ce genre de trompe-l'œil: économie de moyens, suggestion, fascination par des moyens rhétoriques propres à subjuguer l'esprit des générations, et, partant, à engendrer de la tradition? ${ }^{7}$.

Que dit la courte citation du Livre des Mutations annexée à la définition? Il n'est pas inutile de savoir que l'extrait cité est une glose canonique de l'hexagramme kun. Le deuxième des soixante-quatre hexagrammes, kun, représente la Terre, et suit l'hexagramme qian qui représente le Ciel, la Terre figurant en position passive et subordonnée au Ciel, principe souverain et actif. Notre extrait se trouve parmi d'autres exemples indiquant que des changements brusques, heureux ou malheureux, résultent d'une lente accumulation. Les causes du parricide-régicide résultent d'une lente évolution - d'une lente germination, serait-on tenté de dire, pour filer la métaphore de la Terre soumise au Ciel. Si

7. J'ai déjà tenté de définir cette fonction dans Bourgon (1996). 
l'autorité s'effondre d'un coup en apparence, c'est qu'elle a été minée par de multiples «tentatives », pour reprendre l'étymologie suggérée par le Bohutong. Si l'étymologie est douteuse, l'idée est profonde, car le fait que la «tentative», c'est-à-dire l'intention ou le complot non encore réalisés, suffise à constituer le délit, quand bien même sa commission se voit retardée ou déjouée, est le propre de «l'attentat», le crime politique contre l'autorité. ${ }^{8}$

En tant que shi, le meurtre du père est donc conçu comme un complot rampant contre l'autorité. Or, ce crime de shi revêt une importance toute particulière pour la civilisation chinoise. Il s'agit de l'un de ses fondements dogmatiques essentiels, puisqu'il serait la raison d'être des Annales (Chunqiu ou Tch'ouenn Tsi'ou dans l'ancienne transcription usitée dans l'extrait qu'on va lire). Mencius nous apprend en effet que Confucius aurait rédigé ce Classique d'entre les Classiques dans l'intention d'enrayer la décadence des temps, qui se manifestait par une épidémie de régicides-parricides :

Plus tard, les temps devinrent mauvais; la vertu diminua; les fausses doctrines et les anciens désordres reparurent. On vit des sujets mettre à mort leur prince, et des fils ôter la vie à leur père Confucius craignit. [Pour remédier à ce mal] il composa le Tch'ouenn Ts'iou. Le Tch'ouenn Ts'iou rapporte les actions des empereurs, (loue les bonnes, blâme les mauvaises, et enseigne les devoirs d'un souverain). Confucius disait à ce sujet: Ceux qui me connaissent, n'est-ce pas uniquement par le Tch'ouenn Ts'iou qu'ils m'ont connu ? Ceux qui me blâment, n'est-ce pas uniquement à cause du Tch'ouenn Ts'iou qu'ils me blâment ${ }^{9}$ ?

Le Classique, qui aurait été selon Mencius redigé par Confucius lui-même, celui dans lequel il aurait consigné l'essence de son message normatif, est donc avant toute autre chose un dispositif anti-shi, un remède contre l'épidémie de régicides-parricides qui signalent la décadence de la Voie. La pleine signification du terme n'apparaît pourtant qu'à celui qui mesure son ambivalence. Car si le régicide est signe de décadence, il est aussi à l'origine de la civilisation, ou tout au moins de l'ordre étatique sous sa forme dynastique. Dans un article très éclairant, Karine Defoort a montré comment le terme de shi fut l'enjeu d'une bataille terminologique: pouvait-on, devait-on l'appliquer à Tang et à

8. «L'attentat constitue le type même du délit formel, caractérisé sans qu'il soit besoin de constater une atteinte, de déplorer un résultat effectif. Dans l'Iter criminis, il se situe au niveau du commencement d'exécution ou, mieux, lors de l'accomplissement du dernier acte dépendant de l'agent. (Tentative et attentat) : Dans son acception stricte, l'attentat est une tentative érigée en consommation, c'est-à-dire qu'il incrimine comme consommés des actes de tentative, supposant que le but poursuivi par l'auteur de l'attentat a été effectivement atteint ». $C f$. Doucet (2011): «Attentat».

9. Traduction dans Couvreur (1950): 452. 
Wu, c'est-à-dire, respectivement, à celui qui tua l'infâme Gui Jie, le dernier souverain de la dynastie des Xia, et fonda celle des Shang-yin, et celui qui tua le non moins infâme Zhou Xin, dernier souverain des Shang-Yin, pour fonder la dynastie des Zhou? Autrement dit, il s'agissait de légitimer la succession dynastique, en validant une généalogie où la succession régulière des générations naturelles s'émaillait d'actes «contre nature». Nommer Tang et Wu des régicides, c'était légitimer la révolution, ou le «changement de mandat celeste» - on sait que l'une et l'autre sont designés par le même terme : geming. On saisit le sens du débat lorsqu'on sait qu'il avait lieu devant un empereur de la dynastie des Han, dont le fondateur avait renversé et tué le Second empereur de la dynastie des Qin. ${ }^{10}$

À la question: «Ces deux fondateurs étaient-ils des régicides?», deux réponses sont possibles. Celle de Mencius consiste à souligner que la souveraineté du monarque n'appartient pas à sa personne, mais est une fonction qui dépend de l'aptitude du monarque à l'exercer ou, plus exactement, à l'incarner par son exemple moral. Celui qui s'avère indigne de la fonction n'est pas un souverain, mais un simple particulier. Tang et Wu n'ont donc fait l'un et l'autre que tuer chacun un individu privé particulièrement néfaste: ils ne sont pas régicides ${ }^{11}$.

Autre réponse, celle de Dong Zhongshu, qui est le représentant le plus éminent de cette école dite des «textes en caractères modernes» qui, comme indiqué plus haut, privilégie le commentaire de Gongyang et l'interprétation dogmatique des Annales. Tandis que Mencius appréciait le régicide-parricide selon des critères moralistes, en excusant le crime par l'indignité de la victime, Dong Zhongshu fait valoir l'intérêt public et le sens de l'histoire; la légitimité n'est pas affaire de sentiment ou de morale, mais de succession généalogique scellée par un appareillage dogmatique:

Il y a eu soixante-douze hommes dont la vertu était semblable à Yao et Shun [et qui furent donc habilités à succéder à ces deux souverains mythiques]. À présent, vous mettez en doute que l'agression de Tang [contre le dernier des Xia] et celle de $\mathrm{Wu}$ [contre le dernier des Shang-yin] fussent légitimes, mais les soixante-douze souverains ont tous commis une agression. Devrions-nous conclure de vos plaintes que ces soixante-douze souverains sont illégitimes? De fait, quand les Xia on perdu

10. Voir Defoort (1998). J'utilise de préférence la version préliminaire non publiée que l'auteur m'a fort aimablement envoyée.

11. À la question: «Est-il permis à un sujet de tuer son prince?», Meng tzeu répondit: «Celui qui viole la vertu d'humanité, s'appelle malfaiteur; celui qui viole la justice, s'appelle scélérat. Un malfaiteur, un scélérat (eût-il le titre de roi) n'est qu'un simple particulier. J'ai entendu dire que Ou wang punit de mort Tcheou [qui devait être traité comme] un simple particulier; je n'ai pas entendu dire qu'il eût tué son prince». Voir Couvreur (1950): 341 . 
la Voie, les [Shang]-Yin les ont agressés, quand ces derniers ont perdu la Voie, les Zhou les ont agressés, quand les Zhou ont perdu la Voie, les Qin les ont agressés, quand les Qin ont perdu la Voie, les Han les ont agressés. Ceux qui ont la voie agressent ceux qui ne l'ont plus, telle est la règle du Ciel. Il en a toujours été ainsi, ce n'est pas particulier à Tang et à Wu. Ainsi, ceux qui les blâment d'avoir agressé Jie et Zhou doivent aussi blâmer les Qin d'avoir agressé les Zhou et blâmer les Han d'avoir agressé les Qin ${ }^{12}$.

Rhétorique implacable qui mène le contradicteur de la simple spéculation théorique sur la légitimité d'un méfait ancien vers le soupçon de lèse-majesté, aux terribles conséquences pénales. Car, bien sûr, blâmer les Han d'avoir renversé les Qin est un crime politique majeur, passible des derniers supplices. Le plus étonnant est cette ruse de la dogmatique qui transforme un cycle d'usurpations en principe de succession légitime, par le biais du mandat céleste passant d'une dynastie à l'autre: la révolution permanente au principe de la continuité de l'État, il fallait y penser ${ }^{13}$ ! Peu importe le moyen, pourrait-on dire, du moment qu'est enclenché un cycle généalogique de filiation d'une dynastie à l'autre.

\section{Du régicide au parricide: la question de l'intentionnalité}

Mais il s'agit là de régicide, pas de parricide, dira-t-on? Il est vrai que dans les Annales, le premier terme tend à recouvrir complètement le second. Selon Carine Defoort, les vingt-cinq cas de shi cités dans les Annales sont tous des régicides, dont quelques-uns seulement sont aussi des parricides, tandis qu'aucun parricide n'est cité qui ne soit pas aussi un régicide : «il semble que le contexte politique domine le familial», conclut-elle, ce qui l'autorise à traduire shi par «régicide», en négligeant le parricide ${ }^{14}$.

En cette matière, pourtant, le familial est bien difficile à dégager du politique, comme le montrent les jugements où le même Dong Zhongshu statue non plus sur des cas de lèse-majesté ou de légitimité dynastique, mais sur des crimes familiaux. C'est alors qu'il était retiré de la vie publique qu'il rendit ces

12. D'après la citation du Chunqiu fanlu, 25, dans Defoort (1998): 4.

13. On sait que le terme qui désignait dans la Chine impériale ce changement du mandat céleste d'une dynastie à l'autre, geming 革命, a été recyclé pour désigner la révolution dans le monde moderne.

14. «Although shi combines regicide and parricide, the twenty-five cases in the Annals are all instances of regicide, and some of both. In other words, some are regicide but not parricide, none are parricide but not regicide: there is no record of a son simply committing parricide in a merely familial context. Because the political concern seems to dominate the familial one, and for the sake of simplicity, I translate shi as "regicide" only.» Defoort (1998): 8. 
Jugements d'après les Annales sur des cas qui lui auraient été soumis comme à un jurisconsulte privé. Sur les 279 cas qu'aurait compté l'ouvrage original, six seulement nous sont parvenus. Trois d'entre eux portent sur la relation père-fils, dont deux sont des parricides, bien que le père soit battu mais non tué. Nous laisserons de côté le quasi-parricide dont le thème majeur est en fait l'adoption, pour nous concentrer sur celui qui illustre l'unité structurale du parricide et du régicide ${ }^{15}$.

Il convient de noter au préalable que dans ces deux cas de quasi-parricides, les accusés sont, au titre de la loi en vigueur, passibles de la peine de mort la plus infamante: la décapitation avec exposition de la tête. La méthode de Dong Zhongshu consiste à éviter la peine en requalifiant l'acte et, par voie de conséquence, en donnant une nouvelle interprétation de la loi. De même que Tang et $\mathrm{Wu}$ avaient bel et bien tué le souverain régnant, et semblaient mériter la qualification de régicide, les actes décrits dans les cas suivants ont de bonnes raisons d'être appelés parricides. Certes, dans les deux cas, le père n'est pas mort, il n'est que blessé, ou battu, mais s'agissant de coups assénés par un fils sur son père, le code est formel : l'intention suffit à constituer le parricide, l'attentat contre l'autorité est déjà parfait dès lors qu'est commis un acte qui peut être interprété comme sa «tentative». Les auteurs des coups sont donc passibles de la peine du parricide au moment où le jurisconsulte Dong Zhongshu émet le verdict qui va désamorcer le mécanisme du shi.

Blesser son père par erreur

$\mathrm{A}$ [voyant que son] père, $\mathrm{B}$, et $\mathrm{C}$ s'affrontaient en parole, puis en venaient aux mains, et que C s'était saisi d'un sabre pour blesser B, il prit un bâton pour en frapper C, mais il blessa [son père] B par erreur. De quoi A est-il passible?

Quelqu'un dit: «C'est [le crime qualifié par la loi] "battre son père", passible [de décapitation et] d'exposition de la tête.»

Réponse [de Dong Zhongshu] : «Votre humble serviteur croit modestement que père et fils ont une relation des plus proches, et qu'au récit de tels faits, on ne peut ressentir qu'une amère tristesse. Toutefois, en saisissant un bâton pour porter secours [à son père], [A] ne cherchait nullement à l'outrager. Selon le sens des Annales, "Xu Zhi, son père étant malade, présenta un médicament à son père, qui mourut": l'homme de bien (Confucius) se fonde sur l'intention pour pardonner au lieu d'incriminer. [A] ne peut tomber sous le coup de ce que la loi qualifie "battre son père", et ne peut donc être incriminé ${ }^{16}$.»

15. Sur les cas jugés par Dong Zhongshu, voir Huang (1992), Sanft (s.d.) et Bourgon (1996).

16. Cas $n^{\circ} 2$, cité et commenté dans Huang (1992): 37-38. Voir Sanft (s.d.): 13-15. 
Bien que le terme shi n'apparaisse pas dans ce jugement, c'est bien l'étalon sur lequel est évaluée la culpabilité de A vis-à-vis de son père B. C'est le sens du recours à la citation des Annales, où Xu Zhi voulant soigner son souverain-etpère Mai lui fait ingurgiter une médecine fatale, ce qui devait rester comme le cas typique du shi involontaire. Cela ne protégea pas le présumé coupable d'un sort funeste: les Annales nous apprennent qu'il s'enfuit dans la principauté de Jin où il mourut avant que l'année fût écoulée. Les funérailles du père-souverain qu'il avait tué avaient déjà eu lieu, ce que les Annales mentionnent en usant du terme zang, qui désigne les funérailles régulières s'inscrivant dans un ordre de succession légitime. Or, ce terme ne peut être utilisé en cas de shi tant que l'auteur du régicide n'a pas été exécuté. Il y a donc une contradiction dans les Annales, qui qualifient l'acte commis par Xu Zhi de régicide, mais emploient ensuite pour désigner les funérailles un terme qui suppose que le régicide a été expié, alors que son auteur est encore vivant.

Les trois commentaires canoniques des Annales expliquent cette contradiction de manière différente. En tant que chef de file des «textes en caractères modernes », Dong choisit la méthode du commentaire attribué à Gongyang Gao, dont la spécialité consiste à voir dans les incohérences des Annales des jugements sciemment disposés par Confucius à l'intention des initiés. Dans le cas présent, si Confucius a d'abord utilisé le terme shi, c'est pour souligner qu'il y avait bien régicide de fait; s'il a ensuite utilisé le terme de zang désignant les funérailles régulières, c'est pour souligner plus fortement encore que l'absence d'intention rendait le crime excusable en droit. Le droit requalifiant le fait, il n'y a, en définitive, pas eu de shi.

Notons en passant que le rapport entre régicide $s h i$ et funérailles zang est une ébauche antique du devoir de vengeance qui structure à toutes époques le rapport père-fils. Le commentaire Gongyang le résume d'une phrase lapidaire: «Un fils ne peut vivre sous le même ciel que l'assassin de son père »; de même, des sujets loyaux ne peuvent célébrer les funérailles d'un souverain tant que son assassin n'est pas puni. Ne pas venger la mort du père ou du souverain, ne pas tuer le régicide-parricide, c'est s'en rendre complice c'est, en définitive, en être soi-même coupable, conséquence logique dans cet univers de haute densité dogmatique. Inversement, tuer le meurtrier de son souverain n'est pas un meurtre, c'est une reddition de compte, l'annulation d'une dette. Par cette ligne de raisonnement, la logique du shi se fraye un chemin au creux de toutes les législations qui, jusqu'à la fin de l'empire, tolèrent et protègent le vengeur de son père.

Il est certes remarquable que, pour reprendre les termes employés par Dong dans son jugement, «l'homme de bien (Confucius) se fonde sur l'intention pour pardonner au lieu d'incriminer». Que l'absence d'intention mauvaise fût une 
excuse légale était un principe communément admis et clairement énoncé dans les textes canoniques ${ }^{17}$. Mais le régicide-parricide était justement le crime qui ne pouvait être excusé ainsi, tout acte étant nécessairement interprété comme une «tentative» pénétrée d'intention mauvaise. C'est pour cette raison que la loi des Han punit le fait de «frapper son père» du dernier supplice, qu'il y ait eu ou non mort d'homme, qu'il y ait eu ou non intention de nuire. Il est non moins remarquable que pour desserrer l'étau punitif que la loi positive a disposé autour du crime de parricide, Dong Zhongshu doive employer le schème du régicide tel qu'on le rencontre dans l'interprétation que Gongyang fait du passage des Annales. Bien qu'il n'y ait pas régicide, puisque B n'est pas roi, et qu'il n'y ait même pas vraiment parricide, puisque B n'est pas mort, c'est bien sur le patron de shi que Dong Zhongshu a taillé son cas. Il faut en effet que cet acte soit un régicide pour que la maladresse de A soit considérée comme une «tentative», présumant l'intention mauvaise; et il faut cette première phase pour qu'intervienne ensuite, comme un coup de théâtre dogmatique, le pardon de la faute. Où l'on voit que dans ce cas censé mettre en présence un fils et son père, tout est réglé sur le canevas du régicide.

La prévalence du contexte politique sur le familial qui amenait Defoort à traduire shi par régicide en laissant de côté le parricide est une simplification légitime pour un auteur qui décide de ne s'intéresser qu'à l'aspect politique. En vérité, pour autant qu'on puisse juger d'après les gloses classiques et les jugements qui font autorité, cette prévalence du politique fait disparaître le parricide, qui n'est plus qu'un régicide parmi d'autres - un régicide parfait serait-on tenté de dire. En effet, si tout régicide n'est pas forcément un parricide, tout parricide est conçu sur le modèle du régicide: attentat sur une autorité publique, qu'une simple tentative ou une maladresse suffit à constituer en crime d'entre les crimes. La figure du père s'efface ainsi complètement derrière celle du souverain. Et la notion de régicide continuera à infecter celle de parricide durant toute la durée du droit impérial.

\section{Parricide et régicide dans les droits positifs occidental et chinois}

L'assimilation du parricide au régicide n'est certes pas spécifique à la Chine. La tradition juridique occidentale nous en offre divers exemples. Mais à plus ample examen, les ressemblances s'avèrent superficielles, et l'originalité chinoise d'autant plus manifeste.

17. Voir notamment le chapitre des «châtiments de Lü» (Lüxing) dans le Livre des documents. Cf. Shujing: 27.14 . 
Ainsi, à Rome, les Ides de Mars, jour de l'assassinat de César, furent déclarées «jour parricide». Le terme revient ensuite régulièrement pour désigner l'assassinat de divers empereurs, ceux-ci fussent-ils des tyrans aussi impopulaires que Néron ${ }^{18}$. Le terme était devenu si galvaudé dans l'empire tardif que des politiciens sénatoriaux pouvaient traiter de «parricides» aussi facilement que de concussionnaires ou d'adultères, ce dont les auteurs comiques se délectaient ${ }^{19}$. Pour courant qu'il fût devenu, le rapprochement du parricide et du régicide tenait davantage de l'artifice rhétorique que d'une véritable assimilation dogmatique - de même que traiter un chauffard d'assassin n'équivaut pas à l'accuser de meurtre devant la justice. Plus lourd de conséquences serait l'avis donné par un juriste à l'empereur Marc Aurèle que des conjurés qui avaient tenté de le tuer devaient être condamnés à la peine du sac, jusqu'ici réservée au parricide. Mais on ne sait si l'avis fut suivi et la peine appliquée, l'élément le plus sûr étant que l'empereur y était personnellement opposé. En tout état de cause, il s'agit là d'un cas unique, et son issue incertaine montre que l'assimilation régicide-parricide n'avait pas acquis le statut d'une évidence dogmatique. Rien ne rappelle donc en droit romain l'identité profonde du régicide et du parricide telle que le chinois l'a scellée dans le même terme shi.

Qui plus est, alors que le régicide et le parricide sont très tôt associés en Chine, en vertu d'autorités canoniques, cette association est tardive et incertaine en droit romain. Elle apparaît à la toute fin de la République, alors que se profile le régime impérial dont elle est un signe annonciateur : c'est lorsque l'empereur est présenté comme «père de la patrie » qu'attenter à ses jours peut être qualifié de parricide ${ }^{20}$. Comme le note Yan Thomas, «il est difficile d'apprécier le degré de précision juridique d'une telle qualification ${ }^{21} \gg$. La comparaison est une ruse de la propagande impériale, qui n'a guère de conséquences juridiques si l'on excepte le cas jugé sous Marc Aurèle, dont nous avons vu qu'il était douteux. C'est qu'en fait le parricide a une double face, comme sa victime, le pater familias. Le meurtre d'un père était à Rome un crime public, c'est-à-dire l'un de ceux jugés assez graves pour que les autorités publiques se substituassent à la parentèle de la victime, qui était ordinairement chargée de la venger en poursuivant le coupable. S'agissant des crimes et délits banals entre parents, le pater familias avait tout pouvoir pour châtier les fautes commises par les membres de sa domus, s'agît-il d'un domestique ou d'un de ses enfants. Il en découle logiquement que la tentative de parricide était laissée à son jugement:

\footnotetext{
18. Thomas (1981): 693.

19. Ibid.: 689.

20. «En même temps que, à l'époque tardo-républicaine, apparaît et se développe la métaphore juridique du pater patriae, la figure du parricide tend à désigner hyperboliquement les criminels d'État». Voir Thomas (1981): 695.
}

21. Ibid. 
le pater familias décidait souverainement du châtiment de ceux qui avaient attenté à ses jours. En d'autres termes, le parricide était un crime privé, une affaire familiale, tant qu'il restait limité à la tentative. C'est seulement quand la tentative réussissait que, le chef de famille n'étant plus en mesure de châtier ses meurtriers, l'autorité publique s'y substituait ${ }^{22}$. Romains et Chinois procèdent donc de manière diamétralement opposée. Les premiers conçoivent le parricide comme une affaire familiale, qui ne devient un crime public que parce que le chef de famille n'est plus capable d'en faire justice lui-même; et c'est par une extension tardive, alors que l'idéologie impériale cherche à établir le souverain comme «père de la patrie», que le crime de régicide emprunte certains de ces traits du parricide. En Chine, au contraire, la doctrine voit dans le Classique le plus investi d'autorité normative un dispositif contre une épidémie de régicides, qui englobent des cas de parricides. C'est selon le modèle de l'attentat contre la figure royale que porter la main sur son père, même involontairement, même pour son bien, est conçu comme «tentative», présumant l'intention criminelle, punie du pire supplice. Crime familial devenu cause publique à Rome, le parricide est d'abord en Chine un crime contre l'Autorité publique transposé dans le microcosme familial.

La différence observée dans la doctrine se reproduit si l'on examine à présent les peines prévues contre les criminels coupables de régicide ou de parricide. Alors que dans la tradition romaine, puis européenne, les deux crimes étaient punis de peines différentes, ils étaient en Chine punis de la même peine, aussi loin que les codes conservés permettent de remonter.

À Rome, le châtiment du parricide était la peine du sac, dont Cicéron a bien résumé l'esprit:

Comme nos anciens se rendaient compte qu'il n'est rien de si sacré que l'audace ne puisse un jour violer, ils imaginèrent contre les parricides un supplice d'un caractère spécial, capable d'éloigner du crime par l'énormité du châtiment ceux que la nature elle-même n'aurait pu retenir dans le devoir: ils voulurent que les parricides fussent cousus vivants dans un sac de cuir et jetés ainsi dans le fleuve.

Quel unique exemple de sagesse, Ô Juges! N'est-ce pas comme s'ils avaient exclu et arraché de la nature entière cet homme à qui subitement ils enlèvent à la fois le ciel, le soleil, l'eau et la terre, de telle sorte que celui qui aurait donné la mort à celui dont il avait reçu la naissance se trouverait privé de tous les éléments dont tout ce qui existe a, dit-on, reçu la naissance ${ }^{23}$ ?

22. Ibid.: 689.

23. Cf. Cicéron (1921) : 88-89. 
La peine prévue pour le meurtrier de «celui dont il avait reçu la naissance» n'était pas la plus douloureuse, ni la plus spectaculaire: elle était spécialement conçue pour éviter que le criminel «contre nature» ne contaminât par son contact l'un des éléments cosmiques. Davantage que le châtiment d'un crime, il s'agit d'un rituel d'expulsion, susceptible de s'appliquer à d'autres créatures réputées monstrueuses, comme les hermaphrodites. La pudeur romaine tendait plutôt à taire de tels cas, et l'attitude des juges exprime plutôt l'embarras et la honte qu'un acharnement à punir. Au contraire, le complot contre l'empereur était le modèle du crimen majestatis, qualification légale qui entraînait un déploiement extraordinaire de moyens de rétorsion: aveux forcés sous la torture, accusations en chaîne, supplices usant de tous les raffinements de la mort lente, par le feu, par les bêtes, par la croix. Cette différence se retrouve dans l'Europe d'Ancien Régime, où le régicide et sa tentative étaient punis du supplice de l'écartèlement. La peine atroce était un élément d'un dispositif de terreur fort proche de celui qui accompagne le démembrement chinois: le soupçon systématique, la nondénonciation de l'attentat même par un parent proche étant punie comme une complicité, la continuation des poursuites après la mort des coupables, leur corps pouvant être supplicié à titre posthume; la destruction systématique de toute trace du criminel: confiscation du patrimoine, exil de la famille, arasement des maisons ou châteaux, coupe des futaies et interdiction aux collatéraux de porter le nom honni ${ }^{24}$. Ce dispositif de terreur était réservé aux régicides, et de ce fait très rarement employé: deux cas en France au XVII ${ }^{\mathrm{e}}$ siècle - Jacques Clément et Ravaillac - , un seul au XVIII - le fameux Damiens -; une vingtaine de cas, peutêtre, dans toute l'Europe. En revanche, nulle peine n'était fixée pour le parricide, les juges étaient libres de sévir à proportion de l'horreur que leur inspiraient les circonstances et la personnalité de l'accusé. Aucun d'entre eux ne semble avoir eu l'idée de soumettre un parricide à la peine du régicide. Curieusement, c'est la Révolution française qui confondit les deux peines: au titre du code pénal de 1791, puis du code napoléonien de 1810 , le parricide devait «être conduit sur le lieu de l'exécution, en chemise, nu-pieds, et la tête couverte d'un voile noir» pour avoir «le poing droit coupé, et [être] immédiatement exécuté à mort» par décapitation ${ }^{25}$. L'écartèlement ayant été aboli avec les autres supplices de l'Ancien Régime, le code pénal de 1810 stipulait que: «L'attentat ou le complot contre la vie ou contre la personne de l'Empereur est crime de lèse-majesté; ce crime est puni comme parricide, et emporte de plus la confiscation des biens ${ }^{26}$.» Les terroristes qui avaient tenté d'assassiner Louis-Philippe ou Napoléon III se virent

24. Carbasse (1990): 254-255.

25. Code des délits et des peines de 1791 ; code pénal de 1810.

26. Code pénal de 1810 : article 86 . 
donc appliquer la peine du parricide. Mais, là encore, les cas se comptent sur les doigts d'une main, et cet étrange retour du «père de la patrie» romain disparut avec les derniers vestiges du régime monarchique.

Qu'on pardonne cette abondance de détails, qui n'a pour fonction que de bien faire ressortir l'originalité chinoise: du code des Tang à celui des Qing, $\mathrm{du} \mathrm{VI}^{\mathrm{e}}$ au Xx $\mathrm{Xx}^{\mathrm{e}}$ siècle, le régicide et le parricide ont été punis de la même peine: la décapitation avec exposition de la tête sous les Tang, puis le démembrement ou lingchi à partir des Yuan et des Ming - du XIve siècle jusqu'à l'abolition de ce supplice en 1905. Ils ont été poursuivis dans le même esprit et selon la même procédure, initialement prévue pour le régicide, ou plus généralement le complot contre l'autorité publique, la sédition généralisée. Et ceci non quelques fois, au hasard d'analogies douteuses et éphémères, mais systématiquement, en vertu de la loi, dans des milliers de cas au fil des siècles. On a l'impression qu'une machine infernale prévue pour réprimer les grands crimes politiques a fait irruption dans les rapports familiaux : l'attentat régicide reste le modèle sur lequel est conçu le parricide, mais celui-ci ne se limite plus à la relation pèrefils, il couvre l'ensemble des complexes relations hiérarchiques qui constituent la famille chinoise.

\section{Un parricide parmi d'autres : la non-spécificité du meurtre du père dans le droit pénal chinois}

Parvenus au moment de considérer les cas pratiques de meurtres entre parents, nous voyons le parricide se dissoudre dans la parentèle. C'est une caractéristique qui a frappé Françoise Lauwaert, lorsqu'elle dit avoir été surprise «de l'extraordinaire extension donnée au parricide au sens large», dans une «société où s'exprimait une forme d'intégrisme paternel», au sens où le père représentait «l'autorité absolue» pour sa femme comme pour ses enfants ${ }^{27}$. La surprise est légitime, car il y a bien une contradiction: si l'autorité paternelle était si absolue, il semblerait logique que le parricide fût prioritairement, si ce n'est exclusivement, défini comme meurtre du père. Or, ce qui ressort de l'extension en effet extraordinaire du parricide dans le code chinois, c'est justement la non-singularité du meurtre du père, l'effacement de la figure paternelle dans la masse de la parentèle.

Le terme parricide est-il approprié, d'ailleurs? Dans la tradition que nous ont léguée les Romains, le parricide est, fondamentalement, le meurtre «de celui

27. Lauwaert (1999): 27. 
qui a donné la vie», pour reprendre les termes de Cicéron: le père, et seulement lui ${ }^{28}$. Yan Thomas a fermement établi cette lecture, en dépit d'apparences contraires, dont la plus notable est la lex Pompeia de paricidiis, qui étend la qualification de parricide «à tous les meurtres commis sur un parent ou allié, en ligne directe ou collatérale ${ }^{29} »$. Pourtant, indique Yan Thomas, l'extension de cette qualification légale est tardive, l'usage du terme ne la confirme pas. «Parricida-parricidum se disent essentiellement du meurtrier, du meurtre du père - l'agent coupable étant en général le fils et, dans deux cas seulement, la fille $^{30}$.» Le parricide romain, avant comme après la loi Pompeia, reste le meurtre d'un parens, par une extension limitée du meurtre du père. Il en va de même dans la France de l'Ancien Régime, où le parricide est «le meurtre commis dans la personne du père ou de la mère légitimes ou naturels, ou de tout autre ascendant légitime du coupable», pour reprendre l'expression consacrée par les codes révolutionnaire de 1791 et impérial de $1810^{31}$. Le parricide est donc limité à la cellule familiale nucléaire, le père restant le vecteur d'une autorité qui se diffuse à la mère ou, en cas d'absence des parents naturels, à ceux qui en font office (parents adoptifs, tuteurs et autres «ascendants légitimes»). La définition du crime dans le droit pénal actuel est: «Le parricide est le meurtre commis sur un père naturel ou un père adoptif, voire sur un aïeul, par son enfant ou son petit-enfant ${ }^{32}$.» En résumé, le parricide est le crime contre celui ou celle qui exerce l'autorité paternelle.

Ce parricide-là est introuvable dans le droit chinois, qui ne semble avoir connu à toutes époques que le régime de la lex Pompeia: un crime, couvrant toute la parenté proche et lointaine. En fait, l'interdit ne semble pas procéder depuis la place du père, figure d'autorité centrale à partir de laquelle il se diffuserait de proche en proche aux autres parents de sa lignée et, de manière plus limitée, aux parents par alliance. Au lieu de rayonner à partir du noyau paternel, l'autorité semble plutôt descendre des ancêtres les plus éloignés vers les ascendants les plus proches. C'est ce qui ressort clairement de la définition la plus générale qui se trouve dans la section des «règles et définitions » fondamentales, la première dans tous les codes des diverses dynasties des Tang aux Qing. L'article deux de ces codes énumère «Dix infamies», qui ont pour caractéristique commune d'être irrémissibles, c'est-à-dire qu'elles sont exclues des amnisties régulièrement

28. Selon les théories de l'Antiquité européenne dont Aristote est le plus éminent représentant, seule la semence masculine engendre, la femme n'étant qu'un réceptacle germinateur.

29. Le cercle du parricide défini par cette loi est des plus larges puisque, outre les oncles, tantes, parents de l'épouse, il englobe même le patron d'un client; $c f$. Thomas (1981): 651.

30. Thomas (1981): 679.

31. Code pénal de 1791: article 10.

32. «Parricide», dans Doucet (2011). 
décrétées par les empereurs ${ }^{33}$. Le commentaire le plus autorisé du code des Qing explique ainsi cette spécificité:

Selon les Fonctionnaires des Zhou, «Les jugements dans les procès pénaux doivent être fondés sur les relations de parenté entre le père et le fils, et le devoir entre le souverain et son féal». Et aussi «Lorsqu'on applique les Cinq peines, il faut tenir compte des subordinations naturelles (tianlun 天倫)». (Les [dix infamies] qu'énumère cet article sont tous des reniements de la souveraineté et de la parenté (wujun wuqin), des révoltes contre la subordination et des désordres dans les mœurs que le Ciel ni la Terre ne peuvent tolérer et qui révoltent les dieux comme les hommes. C'est pourquoi ils sont exposés en exergue, afin d'alerter les générations ${ }^{34}$.

Par le biais d'une référence classique, nous retrouvons à l'entrée du code pénal les traits fondamentaux du shi, du «parricide régicide ${ }^{35}{ }$. On se souvient que la notice du Bohutong établissait la même analogie de position entre le souverain et le père, entre le serviteur et le fils, en vertu d'un même principe de subordination reproduisant l'ordre cosmique soumettant la Terre au Ciel. Voilà qui fait du père, le délégué du Ciel, le substitut du souverain dans l'ordre familial. Mais s'agit-il bien du père lui-même, d'un individu réel dont la loi viendrait soutenir l'autorité en menaçant ceux qui s'y opposeraient? Ne s'agirait-il pas plutôt de la subordination père-fils, formule élémentaire d'une chaîne de subordination qui traverse de haut en bas toute la parentèle? Cette place est celle de tout ascendant vis-à-vis d'un descendant, indépendamment de toute volonté actuelle, de tout pouvoir effectif. C'est ce que semble indiquer la partie de cet article qui porte spécifiquement sur le parricide, à savoir la quatrième des dix infamies, la «révolte infâme» eni. Elle consiste dans le fait de «frapper ou comploter la mort» de toute une série de parents que l'indétermination du sujet, fréquente dans les textes chinois anciens, rend difficile à restituer de manière aussi concise que l'original. Sont énumérés d'une seule traite: le grand-père ou la grand -mère [paternels], le père ou la mère; puis, pour une épouse, les mêmes individus, à savoir l'un des grands-parents, le père ou la mère de son mari ; pour quiconque, à présent, le fait de tuer un oncle paternel (un frère aîné ou cadet du père) ou l'épouse d'un oncle paternel, une tante paternelle (sœur du père); un frère aîné ou une sœur aînée; l'un des grands-parents du côté maternel; enfin, pour une épouse, tuer son propre mari ${ }^{36}$.

33. Shi'e : littéralement «les dix maux», souvent traduit par «dix abominations ».

34. Shen Zhiqi: 8.

35. Je cite ici le texte Zhouguan 周官, «Fonctionnaires des Zhou», inséré dans le Classique des Documents (Shujing 書經), dont il constitue le chapitre 48.

36. 四曰：惡逆 [謂毆,及謀殺祖父母父母夫之祖父母父母; 殺伯叔父母姑兄姊外祖 父母及者]. 
Voici donc mis sur le même plan un grand nombre de parents, avec une asymétrie qu'a bien signalée Françoise Lauwaert ${ }^{37}$. Ainsi, les coups ou le complot de meurtre du mari par son épouse est une «révolte infâme», mais pas la réciproque, car la relation époux-épouse est calquée sur la subordination du serviteur au souverain, du père au fils. Cette inégalité entre mari et femme retentit sur le statut de leurs parents respectifs: l'épouse qui tue ou blesse l'un des parents de son mari en ligne directe commet une «révolte infâme», mais le mari qui tue l'un des parents de sa femme ne commet qu'un crime ordinaire ${ }^{38}$. Asymétrie que Lauwaert, en disciple de Françoise Héritier, analyse comme la conséquence d'une inégalité fondamentale et constitutive: celle des sexes. Pour être indéniable, et choquante dans ses conséquences pénales, l'inégalité des sexes reste cantonnée à cette cellule, celle qui est en charge de perpétuer la généalogie. Partout ailleurs qu'au sein du couple, le sexe n'importe plus : il est aussi grave de frapper son grand-père que sa grand-mère, son père que sa mère, un oncle ou une tante. Plus étonnant, l'énumération se poursuit avec les frères et sœurs aîné( $e$ ) $s$ : le fratricide est un parricide lorsqu'il est commis sur un aîné, qui est comme tel assimilé au père. Tout ceci fait apparaître clairement le principe ordonnateur: non pas la différence des sexes, mais la subordination des plus jeunes vis-à-vis des plus âgés. Le chinois juridique a pour cela un couple d'expressions toutes faites qui confèrent systématiquement au plus âgé un statut supérieur et une préséance sur le plus jeune : zunzhang ou «respectable aîné » et biyou «humble junior ». Le père de famille n'est qu'un maillon dans la chaîne de l'âge et de la respectabilité; il n'est pas le premier, puisque la chaîne commence par les grands-parents, plus exactement par le plus âgé des aïeux encore vivants. Il est lui-même un «humble junior» vis-à-vis non seulement de ses père et mère, mais de tout parent plus âgé, notamment ses frères et sœurs aînés.

Il peut sembler étrange que la relation père-fils invoquée par le commentaire cité puisse s'étendre à tant de gens du clan. C'est le lieu de rappeler la manière dont la langue chinoise construit des concepts abstraits à partir de couples antithétiques. Lorsque nous lisons jun-chen, et $f u$-zi, nous imaginons un roi et son féal, un père et son fils, une relation bornée à ces deux individus. Or, en chinois, daxiao, binôme composé des adjectifs «grand» $(d a)$ et «petit» (xiao), ne désigne pas un objet ou un individu «grand-petit», mais «la taille», notion abstraite que le chinois saisit comme relation de deux extrêmes, comme polarité.

37. Lauwaert (1999): 74-75.

38. Le fait que le père et la mère de l'épouse ne figurent pas dans la liste des parents dont le meurtre constitue un parricide me paraît invalider le cas, d'ailleurs unique, cité par Lauwaert à l'appui de sa démonstration que la gravité du crime est «déterminée par le sexe de l'individu qui fait lien », le criminel ayant dans ce cas tué la mère de sa femme, relation qui ne figure pas dans la liste des parricides: voir p. 75 et 148-149. 
De la même manière, jun-chen, c'est la «souveraineté», concept abstrait saisi comme le comportement réciproque de deux pôles; $f u-z i$ ne désigne pas un père et un fils, mais la subordination et les devoirs qui en découlent, la «pater-filialité» s'il faut à tout prix substantiver. On comprend dès lors que $f u$-zi puisse s'appliquer à toute la parenté, comme principe de subordination générale aux parents d'âge supérieur.

L'article définissant le parricide comme «révolte infâme » est inclus, rappelons-le, dans la partie des «définitions et règles ». Comme tel, il offre une sorte de schéma général que des lois spéciales vont appliquer à des cas spécifiques en les assortissant de sanctions pénales. Dans tous les codes qui nous sont parvenus, ces lois se trouvent dans la section consacrée aux « homicides 》 (renming). Dans le code des Qing, les lois couvrant les formes de parricide consistent essentiellement en la longue énumération des parents déjà évoquée, précédée d'un verbe qualifiant l'acte punissable: «comploter la mort», «frapper», «tuer par inadvertance», « tuer par négligence», termes qui sont aussi courants pour les crimes ordinaires, commis entre gens non apparentés ${ }^{39}$. Cela signifie qu'aucun terme juridique ne qualifie spécifiquement le parricide. Dans le droit positif, dans les lois qui s'appliquent sous forme de sanctions, il n'existe pas de notion comparable au «régicide-parricide» des textes classiques. Cependant, l'identité de ces deux crimes, celui contre l'autorité politique, celui contre l'ordre familial, apparaît en filigrane dans les dispositions prévues contre le meurtre des parents. C'est d'abord le soupçon systématique qui interprète tout acte menaçant l'intégrité physique d'un ascendant comme une «tentative», un attentat déjà consommé d'avoir été simplement conçu; ainsi, c'est bien le «complot de meurtre des grands-parents et parents » que réprime la loi 284 du code des Qing: si le complot a eu «un début d'exécution, - que cela ait provoqué des blessures ou non -: décapitation pour tous». L'intention suffit à constituer le crime, comme dans les complots contre l'empereur, et la loi ne cherche pas à distinguer de coupable principal de ceux qu'il a entraînés, comme il était de règle pour les crimes ordinaires: meneur, complices et témoins qui n'ont pas dénoncé sont «tous décapités». Si le complot aboutissait à la mort d'un des nombreux ascendants énumérés, la peine était alors le démembrement ou lingchi «pour tous».

Réciproquement, l'intention n'était plus déterminante pour qualifier le crime. Tout acte portant atteinte à l'intégrité physique d'un parent était présumé résulter d'une intention mauvaise, même s'il résultait d'une négligence ou d'une erreur.

39. Ce sont respectivement: la loi 284, «Comploter de tuer les grands-parents paternels, les père et mère» (тоиsha zиfuти fuтu); et 319, «Coups portés aux grands-parents paternels, aux père et mère» (ои zиfити fuти). 
La jurisprudence de Dong Zhongshu sur le quasi-parricide involontaire n'avait pas été sans influence, cependant, car des articles spécifiques prévoyaient la prise en compte de la négligence et de l'erreur, ce qui permettait de réduire la peine à la strangulation au lieu du démembrement pour la négligence, à la décapitation immédiate pour l'erreur ${ }^{40}$. La part faite de ce faible adoucissement, la peine de principe sitôt qu'un acte entraînait la mort d'un ascendant restait le ling $c h i^{41}$. Une conséquence particulièrement dramatique était la condamnation au démembrement des homicides commis dans une crise de folie, qui étaient couramment assimilés à des homicides par négligence, c'est-à-dire des actes commis alors que «ni les sens ni l'intelligence ne permettaient d'en prévoir la conséquence $^{42}$ » (еrmu suo buji, silï suo budao). Quelques grâces furent ainsi octroyées à des fous parricides au début des Qing, mais à partir des années 1740, l'excuse de folie ne fut plus prise en compte, et des malades mentaux furent condamnés au démembrement aussi systématiquement que les autres parricides. En conclusion de sa magistrale étude sur le sujet, Luca Gabbiani voit dans cette évolution une «négation des fondements théoriques du droit traditionnel ${ }^{43}{ }^{3}$, ce qui est vrai si l'on ne considère que les principes de la responsabilité pénale établis depuis l'Antiquité pour le droit commun. Mais, précisément, la notion de parricide-régicide appartenait à un autre droit exorbitant du droit commun, celui régissant la famille et la souveraineté impériale, dont les fondements théoriques étaient, hélas, aussi traditionnels.

La parenté du «supplice des cent morceaux» chinois avec les peines européennes qui punissaient le régicide en disloquant le corps, que ce soit l'écartèlement français ou le quartering britannique, est assez évidente pour se passer de démonstration. C'était en Chine aussi la punition typique des grands crimes politiques: la grande rébellion, la haute trahison, le banditisme armé. Comme tel, le lingchi était accompagné de mesures extrêmes comme la responsabilité collective des «trois clans» du coupable: ses parents du côté paternel, maternel, ainsi que ceux de sa femme devaient être selon les cas soit exécutés, soit frappés de peines de déportation ${ }^{44}$. C'est là encore un équivalent aggravé des peines qui frappaient le régicide dans la France d'Ancien Régime. Pour atroce qu'il soit, ce dispositif de terreur avait sa logique: celui qui menaçait l'ordre politique de désagrégation devait être lui-même désagrégé, ainsi que sa famille et tout ce qui

40. Sur les articles du code des Qing 292-11, «Homicide par négligence des grands-parents et parents» (Guoshi sha zufumu fumu), et 319-10, «Homicide par erreur des grandsparents et parents » (Wusha zufumu fumu), voir Gabbiani (2009): 342.

41. Ibid.

42. Gabbiani (2009): 343 et 365 .

43. Gabbiani (2009): 387.

44. Cf. Brook, Bourgon, Blue (2008): chap. 4 en particulier. 
la symbolisait. Employer une telle peine, couramment associée à de tels moyens, pour défendre non plus le chef d'un corps politique, mais un parent ascendant victime d'un autre parent plus jeune, était non seulement cruel mais absurde, car c'était courir le risque de disloquer le clan en prétendant le sauver. Pourtant, les archives judiciaires des Qing montrent que le lingchi fut plus souvent employé contre des enfants parricides ou des femmes qui assassinaient leur mari que pour réprimer des rébellions ou des bandes armées. La néfaste association du régicide et du parricide a donc déterminé l'emploi des mêmes peines pour les crimes politiques et les crimes familiaux bien après que l'association dans les textes antiques entre parricide et régicide se fut estompée.

\section{Une autorité pater-maternelle?}

Le «parricide» chinois, si tant est qu'on puisse encore employer ce terme, n'est donc pas un crime contre l'autorité paternelle; c'est un crime contre la règle de subordination qui englobe tous les parents du lignage. Nous sommes ici très loin de la paternité romaine. De fait, si le meurtre du père n'est pas distingué de celui de tant d'autres parents, c'est que le père chinois n'a aucune des prérogatives que le droit romain accorde au pater familias. Aussi loin que les lois écrites qui nous sont parvenues permettent de remonter, le père chinois n'a jamais eu ce droit absolu de vie et de mort sur sa descendance mâle qui fondait l'autorité sur la terreur ${ }^{45}$. Certes, on sait que, jusqu'au siècle dernier, des clans puissants s'arrogeaient assez fréquemment le droit d'exécuter des fils indignes ou des filles perdues. Mais la pratique ne fut jamais autorisée par la loi, et à bien observer ces pratiques illégales, on constate qu'elles ne résultaient pas du diktat d'un pater familias tout puissant, mais d'une décision prise par un conseil de lignage qui officiait au nom des ancêtres, dont les pères vivants n'étaient que les représentants. D'un point de vue légal, le père ou la mère qui tue son enfant était coupable de meurtre, crime capital, même si le principe de subordination qui régissait le droit familial aboutissait en fait à une réduction de peine. Du point de vue du droit civil, à présent, le parricide est toujours le meurtre de «celui qui a donné la mort à celui dont il aurait reçu la naissance» défini par Cicéron, à ceci près que la naissance n'est plus considérée comme engendrement biologique, mais comme l'institution d'un citoyen par la succession au patrimoine. Si le meurtre du père est «contre nature », c'est qu'il revient à s'instituer soi-même, en usurpant la volonté paternelle censée s'exprimer de manière posthume dans le testament. De nombreuses fictions de la littérature occidentale ont exploité le lien entre parricide et testament où l'on voit le fils

45. Voir sur ce point Thomas (1984). 
usurper le pouvoir paternel, et falsifier ses dernières volontés. Or, la Chine est une civilisation sans testament ${ }^{46}$. La forme légale de succession était la division du patrimoine en parts égales entre les frères, du vivant des parents, et sous la supervision du clan. Les volontés du père n'avaient qu'un rôle marginal visà-vis du principe d'égalité censé présider au partage, la seule exception étant la part supplémentaire accordée au frère aîné chargé de l'entretien des parents et du culte ancestral. De quelque manière qu'on la considère, comme pouvoir de punir ou comme pouvoir de léguer, l'autorité du père chinois est donc très limitée comparée à son homologue occidental. Sa volonté était fermement encadrée par la loi étatique, ainsi que par les règles de fonctionnement du clan qui le plaçaient «à l'ombre des ancêtres ». Il est donc logique que les lois sur le «parricide» aient eu fonction de défendre non l'autorité paternelle, mais la cohérence du clan reposant sur l'obéissance aux aînés.

Il en découle, sur le plan dogmatique auquel nous revenons pour conclure, une curieuse conception de l'autorité. Celle que nous avons héritée des Romains est exclusivement masculine, c'est une extrapolation de l'autorité paternelle. Or, l'autorité chinoise est mixte, féminine autant que masculine, comme le montre l'équilibre ménagé entre la ligne paternelle et la ligne maternelle dans les lois sur le parricide. Loin d'être le fruit du hasard, ou de pratiques inconscientes, il s'agit là d'une construction dogmatique savante, dont la formulation la plus claire se trouve dans le commentaire qui accompagne les lois dans le code des Tang. Ainsi, la quatrième des «Dix infamies », la « révolte infâme » dont nous avons déjà parlé, est rapportée à un terme qui offre un autre équivalent du «parricide», sans doute le plus évocateur sur le plan doctrinal. C'est le binôme jingxiao, un couple d'animaux qui sont, le premier, une «sorte de léopard en plus petit qui, à l'orée de sa vie, commence par dévorer sa mère ${ }^{47}$ »; et le second, la chouette, dont le petit a la même déplorable réputation de dévorer sa mère. Nous voici donc avec deux petits matricides, et pas de parricide au sens strict de «meurtrier de son père ». Mais comme tout s'arrange dans le meilleur des mondes dogmatiques, le petit jing, chaque fois qu'il est associé à xiao, est réputé manger son père et non sa mère, comme s'il se dévouait pour rétablir la parité nécessaire à une saine conception du parricide. Car c'est bien cet équilibre que le commentaire établit, en s'appuyant sur quelques vers du Livre des Odes, l'un des Classiques canoniques ${ }^{48}$ : «Les bienfaits des père-et-mère/Sont hauts

46. Quelques testaments ont été découverts dans les tombes des Han; mais la pratique semble avoir disparu par la suite. Elle est inconnue dans le corpus juridique qui nous a été transmis depuis les Tang.

47. Selon la définition du Shuowen jiezi.

48. Shijing II.5. 8 (Legge, 1878, t. $4: 352$ ). 
comme le ciel et sans limite/Être institués successeurs de nos ancêtres/N'est pas une mission à négliger./ Avoir un cœur de patricide-matricide/C'est anéantir ensemble l'amour et le respect ${ }^{49}{ }^{»}$.

Le commentaire est construit sur la mise en parallèle antithétique des «bienfaits des père-et-mère», (fumu zhi en), avec le «cœur du patricide-matricide», (jingxiao qixin), où nous retrouvons nos deux petits animaux, celui qui mange son père et celui qui mange sa mère. Chaque tronçon du parallélisme ménage soigneusement l'équilibre du couple parental, auquel un autre passage vient apporter une dimension généalogique. «Être institués successeurs de nos ancêtres » (siji bizu), en effet, c'est succéder à une double lignée d'ancêtres en ligne féminine $b i$ et en ligne masculine $z u$. Que les ancêtres de la mère soient cités avant ceux du père, la lignée cognatique avant l'agnatique, ne doit pas être regardé comme un signe de préséance, selon Legge, mais seulement comme une licence de style ${ }^{50}$. C'est possible mais, outre que tout au long du Shijing, la parenté est désignée sous le terme bizu où les cognats $b i$ précèdent les agnats $z u$, nous voyons une autre prévalence de la ligne féminine dans le couple jingxiao, où les deux animaux sont à l'origine matricides, avant que jing ne se dévoue pour manger son père et rétablir ainsi l'équilibre. ${ }^{51}$ Autre bizarrerie : xiao la chouette, dont le petit est réputé manger sa mère, est aussi le nom d'une peine infamante. Depuis les Tang jusqu'à son abolition en 1905, le terme a servi à désigner la pratique consistant à exposer la tête du criminel, ce qui était une forme aggravée de la décapitation. Or, xiao est précisément la peine dont les lois des Han frappaient l'atteinte à l'autorité paternelle, comme nous l'ont montré les jugements de Dong Zhongshu sur deux cas de quasi-parricides. Le symbole du matricide et la peine qui punissait le parricide portaient donc le même nom de xiao, cas d'identité entre la peine et le crime qui suggérerait que le meurtre étalon était à l'origine non celui du père, mais celui de la mère. Il serait facile de glisser de tels constats vers les spéculations de Granet sur les origines matriarcales de la société chinoise. Nous nous bornerons à n'y voir qu'un montage dogmatique justifiant la composition du lignage et contribuant ainsi au relatif effacement du père dans la définition du parricide.

Par une étrange ironie, c'est la civilisation qui exclut le plus radicalement les femmes du pouvoir, au point qu'il n'y eut comme on sait jamais d'impératrices qui ne fussent «douairières », c'est-à-dire des veuves d'empereur, qui

49. Tanglü shuyi, commentaire (shu 疏) de la $4^{\mathrm{e}}$ des «Dix infamies», la «Révolte infâme» (e'ni).

50. «The feminine term is put first for the requirement of the rhyme» (Legge, 1878, t. 4: 304).

51. Notons aussi que le terme se rencontre plus souvent sous la forme xiaojing que sous la forme jingxiao, ce qui revient à placer le meurtre de la mère avant celui du père. 
Jérôme Bourgon

développa une conception mixte de l'autorité. Du fonctionnaire «père-et-mère » fumuguan, qui était l'appellation familière des mandarins, au dragon mâle et au phénix femelle qui symbolisaient le pouvoir impérial, nombreux sont les signes d'une bisexualité du pouvoir qui avait aussi ses aspects fonctionnels ou pratiques : ainsi, en droit pénal, la bienveillance, vertu féminine, devait tempérer la rigueur, et c'était là une conception assez sérieuse pour que des traités de jurisprudence fussent conçus sur ce principe.$^{52}$ Mais l'ironie vient nous frapper en retour: d'avoir été plus souvent dans le passé et d'être à présent légalement exercée par des femmes, la conception occidentale de l'autorité en a-t-elle cessé pour autant d'être masculine?

52. Voir notamment la version amplifiée des «Jugements à l'ombre du Poirier» publiée sous les Ming par Wu Na, où l'on voit des magistrats consulter leur femme ou leur mère sur des cas capitaux épineux; j’y ai fait une brève allusion dans Bourgon (2000). 
Lapsus de Laïus. Entre régicide et parricide, l'introuvable meurtre du père

\section{BibliographIE}

Bourgon Jérôme (1996b). Les vertus juridiques de l'exemple. Nature et fonction de la mise en exemple dans le droit de la Chine impériale. Extrême-Orient, ExtrêmeOccident, $\mathrm{n}^{\circ}$ 19: 7-44.

Bourgon Jérôme, (2000). (Sauver la vie). De la fraude judiciaire en Chine à la fin de l'empire. Actes de la Recherche en Sciences Sociales, $\mathrm{n}^{\circ} 133$, juin, «Les sciences de l'État»: 32-39.

Bohutong, chapitre 99, «Zhufa 誅伐» (châtiments et expéditions punitives), dans l'édition de Tjan Tjoe Som, Bo Hu T'ung, 2 vol. Leyde: Brill, 1949-1952, mise en ligne par l'université de Virginie: http://www2.iath.virginia.edu/saxon/servlet/ SaxonServlet?source=xwomen/texts/

Brook Timothy, Bourgon Jérôme et Blue Gregory (2008). Death by a Thousand Cuts. Harvard University Press.

CARbasse Jean-Marie (1990). Introduction historique au droit pénal. Paris: Presses universitaires de France.

Code pénal du 25 septembre-6 octobre 1791, version en ligne: http://ledroitcriminel. free.fr/la_legislation_criminelle/anciens_textes/code_penal_25_09_1791.htm

Code pénal de 1810, version intégrale telle que mise en ligne: http://ledroitcriminel. free.fr/la_legislation_criminelle/anciens_textes/code_penal_de_1810.htm

Couvreur Séraphin (1950). Les Quatre Livres - IV. Cuvres de Meng Tzeu, Les Humanités d'Extrême-Orient, Cathasia, série culturelle des Hautes Études de Tien-Tsin. Paris: Les Belles Lettres, version numérique fournie en ligne par l'université du Québec à Chicoutimi.

Cić́ron (1921). Pour Sextius Roscius d'Amérie, XXV, 70-XXVI, 71: 88-89, in Discours t. 1, Paris: Les Belles Lettres, «Guillaume Budé».

DEFoort Carine (1998). Naming and Regicide in the Annals, version préliminaire non publiée de «The Rhetorical Power of Naming : The Case of Regicide, Asian Philosophy, $\mathrm{n}^{\circ} 8$ (2): 111-118, mis en ligne: http://dx.doi.org/10.1080/09552369808575476

Doucet Jean-Paul (2011). Dictionnaire de droit criminel, édition en ligne: http:// ledroitcriminel.free.fr/dictionnaire.htm

Gabbiani Luca (2009). Pour en finir avec la barbarie. Folie et parricide en Chine à la fin de l'ère impériale (XvIII'-XX' siècles). T'oung Pao, $\mathrm{n}^{\circ}$ 95: 334-392.

HuANG Yuansheng (1992). Dong Zhongshu Chunqiu zheyu anli yanjiu (Étude sur les précédents dans les Jugements d'après les Annales de Dong Zhongshu). Faxue luncong, $\mathrm{n}^{\circ} 21(2), 29-63$.

Johnson Wallace (trad. et prés.) (1979). The Tang Code. Vol. I. General Principles. Translated with an Introduction by Wallace JoHNSON, Princeton: Princeton University Press.

LAUWAERT Françoise (1999). Le Meurtre en famille. Parricide et infanticide en Chine (XVIII ${ }^{e}$-XIX $X^{e}$ siècle), Paris: Odile Jacob.

LegGe James (1861). Chinese Classics. vol. IV. The Book of Odes, rep. Taipei. 


\section{Jérôme Bourgon}

Magdelain André (1984). Paricidas. In Yan Thomas (dir.), Du châtiment dans la Cité: supplices corporels et peine de mort dans le monde antique. Rome: École française de Rome: 549-571.

SAnFt Charles (s.d.). «Dong Zhongshu's Chunqiu jueyu: Translation and Analysis, with Reference to Recovered Qin and Han Materials », mémoire non publié de l'université de Kyoto.

SHEN Zhiqi 沈之奇, Da Qing lüli jizhu, 大清律輯注, édition moderne en 2 vol. par HuAI Xiaofeng 懷效鋒. Pékin: Falü chubanshe, 1998 (Zhongguo lüxue congkan).

Thomas Yan (1981). Parricidium. Mélanges de l'École française de Rome. Antiquité, t. 93, nº 2: 643-715.

Thomas Yan (1984), «Vitae necisque potestas. Le père, la cité, la mort», in Yan Thomas (dir.), Du châtiment dans la Cité: supplices corporels et peine de mort dans le monde antique. Rome: École française de Rome: 499-548 
Lapsus de Laïus. Entre régicide et parricide, l'introuvable meurtre du père

\section{GlossaIRE}

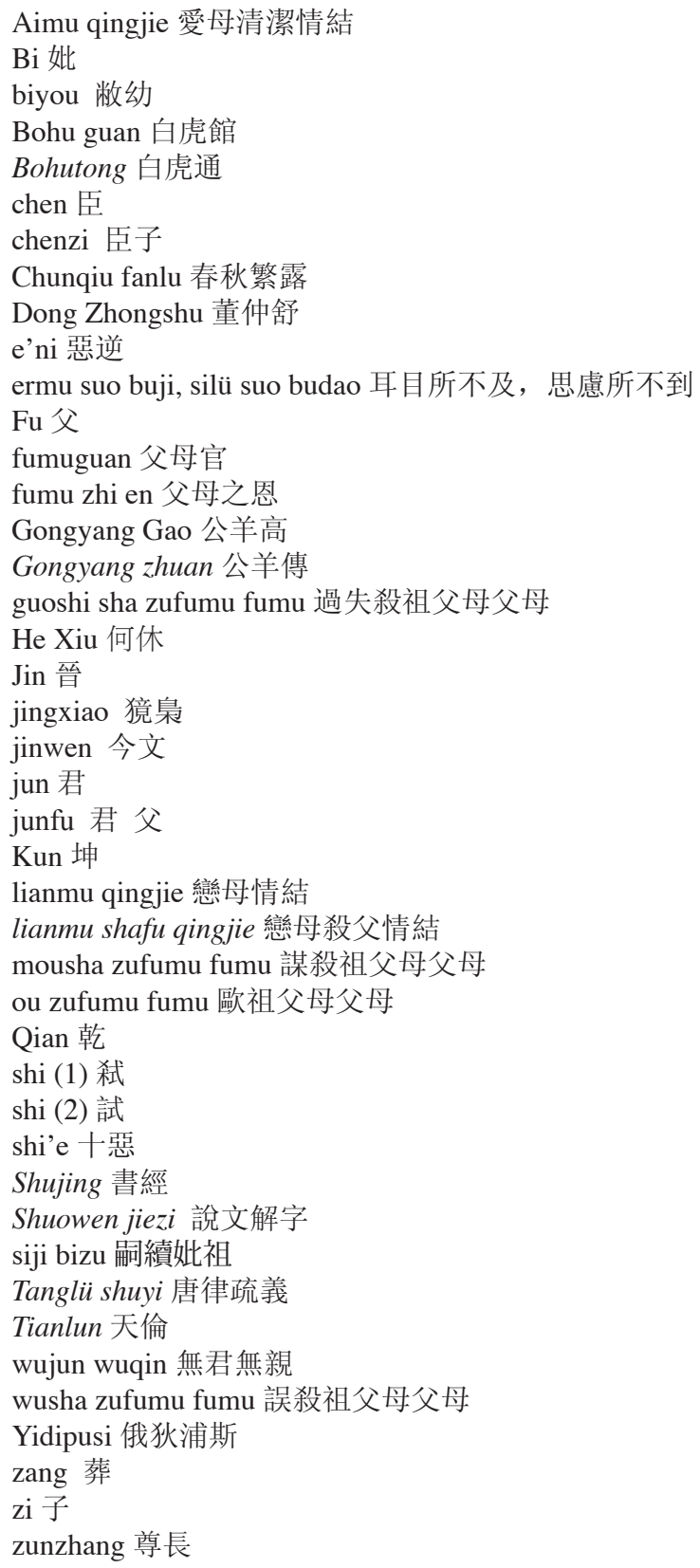


